\title{
PROJETO NOVOS TALENTOS COMO FERRAMENTA DE DEMOCRATIZAÇÃO DO ENSINO
}

\author{
NEW TALENTS PROJECT AS A DEMOCRATIZATION TOOL FOR THE EDUCATION
}

Camila Helena Menezes de Oliveira ${ }^{1}$, Danielle Yumi Mizuno² ${ }^{2}$ Felipe de Melo Medeiros ${ }^{3}$, Hingred Karoline Magalhães da Luz ${ }^{4}$, Rebeca Dias de Souza ${ }^{5}$, Rones de Souza Santos ${ }^{6}$, Adriano Frutuoso da Silva ${ }^{7}$

DOI: 10.37702/REE2236-0158.v39p432-440.2020

\section{RESUMO}

As desigualdades socioeconômicas podem ser constatadas em vários aspectos na sociedade. Um deles é a baixa expectativa, por parte de alunos de escolas públicas brasileiras, de cursar o Ensino Superior. Tendo em vista essa realidade, o grupo PET de Engenharia Civil da Universidade Federal de Roraima (UFRR) criou o projeto Novos Talentos, em 2017, com o intuito de apresentar a universidade pública e o curso de Engenharia Civil às escolas públicas de Roraima, bem como auxiliar na democratização do Ensino Superior. O projeto tem sido desenvolvido desde sua criação e, em 2019, sofreu alterações e passou a englobar os cursos de Engenharia Elétrica e Arquitetura e Urbanismo, em parceria com os centros acadêmicos desses cursos da UFRR. Em 2019, também, o projeto conseguiu atingir novas localidades, como o país fronteiriço Guiana e a comunidade indígena Canauanim. Este artigo tem como objetivo apresentar as ações desenvolvidas dentro do projeto no ano de 2019 e analisar seus impactos, com ênfase nas novas localidades abrangidas. Para isso, observou-se o engajamento e interesse dos alunos nas escolas durante a ação, bem como a abrangência em número de participantes e de locais atendidos. No ano de 2019, o projeto foi apresentado para um total de 419 alunos de escolas em Roraima e na Guiana, totalizando 1465 alunos desde sua criação. Houve um crescimento significativo da ação em 2019, tendo a parceria com os centros acadêmicos contribuído de forma positiva para essa evolução. Ademais, foram estabelecidos importantes vínculos da universidade com escolas e com a comunidade.

Palavras-chave: escolas públicas; acesso ao Ensino Superior; permanência; extensão universitária.

\begin{abstract}
Socioeconomical inequalities can be often found in several spheres within our society. One of them is the low expectations from Brazilian public school students of getting a higher education degree. Based on this reality the Civil Engineering PET group from the Federal University of Roraima (UFRR) created the New Talents project in 2017 in order to share information regarding the public university and the Civil Engineering course in public schools in the state of Roraima and also to contribute to a more democratic access of the higher education. The project has progressed since its beginning and it was modified in 2019, when a partnership with the UFRR's Electrical Engineering and Architecture and Urbanism academic centers was settled, leading to an inclusion of those courses in the project's presentation. The project also succeeded at reaching other locations in 2019, such as the bordering country Guyana and the indigenous community of Canauanim. This paper's goal is to present the actions developed within the project in 2019 and to analyze its results, with an emphasis on the new locations reached. To do this analysis, the involvement and interest of the school students
\end{abstract}

\footnotetext{
${ }^{1}$ Graduanda em Engenharia Civil, Universidade Federal de Roraima; camilamenezes62@gmail.com

${ }^{2}$ Graduanda em Engenharia Civil, Universidade Federal de Roraima; yumimizuno17@gmail.com

${ }^{3}$ Graduando em Engenharia Civil, Universidade Federal de Roraima; felipemedeiros25@ gmail.com

${ }^{4}$ Graduanda em Engenharia Civil, Universidade Federal de Roraima; hingredpss@ gmail.com

${ }^{5}$ Graduanda em Engenharia Civil, Universidade Federal de Roraima; rebecadiassouza@outlook.com

${ }^{6}$ Graduando em Engenharia Civil, Universidade Federal de Roraima; rnsdesouzasantos@gmail.com

${ }^{7}$ Tutor, Doutor em Engenharia Civil, Universidade Federal de Roraima; adriano.silva@ufrr.br
} 
were observed, as well as the numbers of participants and locations assisted. In the year of 2019, the project reached 419 students from schools in Roraima and Guyana, leading to a total of 1465 students since its creation. There was a significant growth of the project in 2019, with a positive contribution from the partnership with the academic centers. Furthermore, important bonds were created between the university, schools, and the community.

Keywords: public schools; higher education access; college completion; university; extension.

\section{INTRODUÇÃO}

Dadas as grandes desigualdades socioeconômicas existentes no Brasil, diversos alunos da rede pública não têm perspectiva de cursar um Ensino Superior, seja por dificuldades financeiras ou por falta de incentivo das escolas em que estão inseridos, fazendo com que optem muitas vezes por uma escolarização que permita a entrada mais rápida no marcado de trabalho (ORTEGA, 2001). Nesse contexto, muitos desses jovens também desconhecem o processo seletivo para o ingresso em universidades, incluindo as etapas, os conteúdos programáticos, a existência de cotas e até mesmo as datas de inscrição dos principais vestibulares (SAMPAIO, 2011).

Por outro lado, mesmo para os alunos que conseguem êxito na entrada do Ensino Superior, as consequências advindas dos déficits da educação na rede pública perduram, fazendo com que grande parte dos universitários com esse perfil não concluam seus cursos (FREITAS et al., 2017). Desse modo, embora existam bolsas e auxílios dentro da universidade pública que visam a diminuir os índices de evasão, muitos alunos universitários em estado de vulnerabilidade desconhecem esses programas, o que se mostra desfavorável à permanência no Ensino Superior.

Reverter esse quadro de acesso desigual às Instituições de Ensino Superior (IES) e ainda de evasão acadêmica é uma tarefa complexa, entretanto algumas medidas podem ser tomadas. Diante disso, foi criado o Projeto Novos Talentos, uma iniciativa do Programa de Educação Tutorial (PET) de Engenharia Civil da Universidade Federal de Roraima (UFRR), a fim de levar o curso de Engenharia Civil às escolas públicas do Estado, esclarecendo sobre as áreas da engenharia, as formas de ingresso e as bolsas e auxílios na Universidade.

O projeto é executado desde o ano de 2017, quando o enfoque eram escolas de Ensino Médio da rede pública, situadas na própria capital do estado, Boa Vista. Desde então, a ação foi expandida para outros municípios de Roraima e no ano de 2019 foi levada para a comunidade indígena Canauanim, composta pelos povos Macuxi e Wapichana, distribuídos entre 1338 habitantes. Nesse mesmo ano, a expansão do projeto também possibilitou a ida à escola St Ignatius Highschool na cidade de Lethem - Guiana, país fronteiriço ao estado de Roraima. Ademais, outra mudança ocorrida no ano de 2019 foi a associação do grupo PET Engenharia Civil ao CAAU (Centro Acadêmico de Arquitetura e Urbanismo) e ao CAEEL (Centro Acadêmico de Engenharia Elétrica), ambos da UFRR, passando a levar para essas localidades informações não só da Engenharia Civil, como também de Arquitetura e Urbanismo e Engenharia Elétrica, possibilitando o compartilhamento de relatos mais diversificados acerca da universidade pública.

O projeto, ao abranger áreas como a aldeia indígena Canauanim, mostrou-se extremamente relevante, pois, de acordo com o Censo de 2010, publicado pelo IBGE, 11,0\% da população do estado de Roraima se autodeclara indígena, sendo o estado que detém o maior percentual do Brasil. Em contrapartida, apesar de atualmente existirem programas que visam à inserção desses povos no Ensino Superior, ainda são poucos os que assim o fazem, pois estão muitas vezes isolados em suas comunidades sem que a continuação dos estudos seja lhes apresentada como uma 
realidade possível. É necessário acrescentar que a expansão do Novos Talentos para a comunidade indígena Canauanim possibilitou um grande avanço ao projeto, pois, além de levar todos os conhecimentos de ingresso na universidade, bolsas, auxílios e caracterização dos cursos, também introduziu atividades interativas e dinâmicas em grupo, voltadas ao público infanto-juvenil.

Diante disso, o projeto objetivou atuar como uma medida de democratização do acesso ao Ensino Superior, levando a possibilidade de prosseguimento dos estudos para alunos de escolas públicas, que se encontram em vulnerabilidade social, sobretudo de áreas mais afastadas ou de difícil acesso, promovendo positivamente os cursos de Engenharia Civil, Engenharia Elétrica e Arquitetura e Urbanismo da UFRR, além da instituição em si, nas escolas visitadas. Ademais, o presente artigo se propõe a discutir e a explorar os novos impactos atingidos com a edição 2019 do Novos Talentos, bem como possibilidades futuras de crescimento do projeto.

\section{METODOLOGIA}

Para a realização do projeto, foi necessária uma mobilização iniciada pelo grupo PET Civil -UFRR, que se juntou aos Centros Acadêmicos de Arquitetura e Urbanismo e de Engenharia Elétrica, dois cursos de áreas afins, para expansão do projeto. Depois de duas reuniões para definir como seria a realização do projeto no ano de 2019, os grupos montaram apresentações digitais com informações de seus cursos e áreas profissionais para que fossem explicadas nas escolas. Além disso, foi desenvolvido um conteúdo inicial com informações de ingresso na Universidade no Ensino Superior e sobre auxílios e bolsas oferecidos pela Universidade Federal de Roraima.

Em seguida, os participantes do projeto sugeriram escolas da região periférica de Boa Vista - RR e do interior do estado, com as quais eles conseguiriam entrar em contato para realização do projeto. O planejamento das escolas que seriam atendidas e as datas de execução foram determinados de acordo com a disponibilidade de tempo de cada escola e dos participantes do projeto. Para cada instituição que participou do Novos Talentos, foram enviados dois integrantes de cada grupo para executar a ação, instruídos por alunos que já haviam participado anteriormente.

A partir de sugestões baseadas nos resultados da ação em anos anteriores, o projeto passou a englobar também alunos do Ensino Fundamental. Houve, então, reuniões com os grupos participantes para debater sobre a forma de abordagem, tendo sido decidido o desenvolvimento de atividades dinâmicas e interativas para captar o interesse das crianças e, ao mesmo tempo, fornecer a elas explicações de assuntos relacionados aos cursos. Após isso, cada grupo ficou responsável por desenvolver suas próprias dinâmicas e apresentá-las aos demais.

A ação alcançou a escola da comunidade indígena Canauanim, localizada no município de Cantá - RR, durante sua semana cultural, cujos alunos eram principalmente crianças e jovens, de idades muito variadas. Nessa escola, foram desenvolvidas as dinâmicas preparadas pelos grupos: o grupo PET - Engenharia Civil levou imagens de formas geométricas tridimensionais e pediu que os alunos tentassem montá-las com palitos de madeira e massinha de modelar (Figura 1); o Centro Acadêmico de Arquitetura e Urbanismo levou peças de madeiras que combinadas formavam casas e prédios (Figura 2); e o Centro Acadêmico de Engenharia Elétrica apresentou a mini bobina de Tesla, que consiste em um sistema abastecido por bateria capaz de acender uma lâmpada por indução eletromagnética. As dinâmicas foram realizadas em uma sala de aula, onde as carteiras foram afastadas e todos se sentaram no chão. Os alunos foram divididos em grupos e foi realizado um sistema de rotação para que todos participassem das dinâmicas do PET, CAAU e CAEEL. 
Figura 1 - Figura 3D construída pelos alunos da aldeia indígena Canauanim com palitos de madeira e massinha de modelar

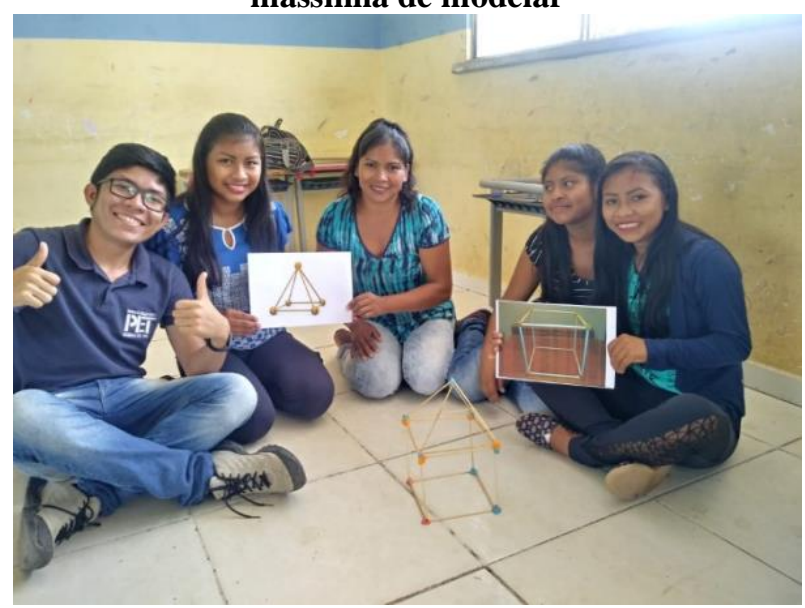

Fonte: arquivo dos autores (2019).

Figura 2 - Dinâmica com blocos de madeira realizada pelo CAAU na comunidade indígena Canauanim

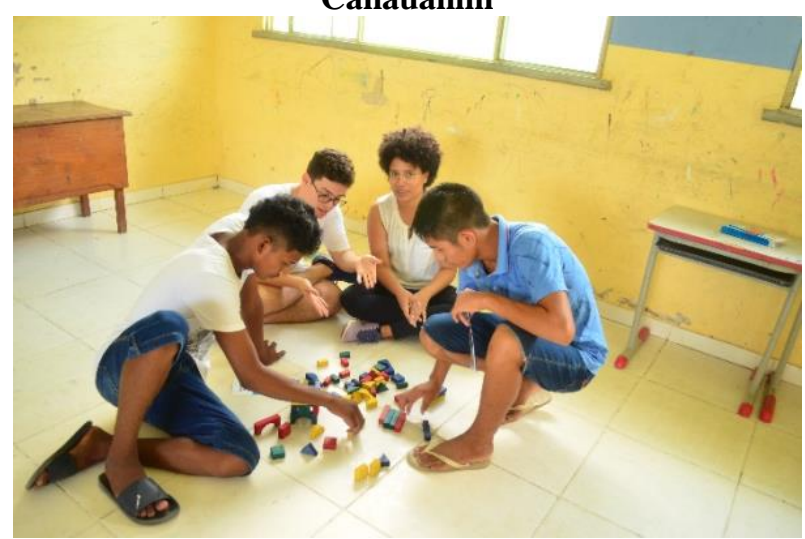

Fonte: arquivo dos autores (2019).

Para a realização da dinâmica proposta pelo PET, foi pedido que os alunos se organizassem no chão em um círculo único para que a dinâmica fosse explicada e, então, o grupo foi dividido em dois ou três subgrupos para que um desafio fosse proposto: o subgrupo que terminasse mais rápido, usando menos palitos, seria o vencedor. Depois que o grupo vencedor foi selecionado, a situação foi utilizada como contexto para se discutir como ocorre na prática a atuação do engenheiro civil. Comentaram que $o$ engenheiro deve trabalhar em equipe e encontrar soluções rápidas e econômicas para o problema proposto e, por isso, era importante que os alunos encontrassem a melhor solução trabalhando juntos. Ainda, para ilustrar as áreas da Engenharia, foram exibidas imagens impressas de obras de construção civil, estradas, estruturas e pontes. Em seguida, foi explicada a íntima relação entre o trabalho do engenheiro e do arquiteto na construção civil, evidenciando a relevância das duas áreas. Após essa dinâmica, que durava cerca de 30 minutos, os grupos do PET, do CAAU e do CAEEL foram rotacionados e a dinâmica iniciada novamente. É importante ressaltar que, ao fim de cada dinâmica, foi comentado com os alunos sobre o processo seletivo indígena, e os grupos buscaram, por meio de diálogo, incentivá-los a participar.

Posteriormente, o projeto foi convidado a participar da XI Feira Intercultural da Escola Estadual Militarizada Aldébaro José Alcântara, envolvendo escolas dos municípios de BonfimRR e Lethem, cidade da Guiana, país fronteiriço ao Brasil, a cerca de $133 \mathrm{~km}$ da capital de Roraima. Nesse evento, houve apresentações de diversos projetos da Universidade Federal de Roraima bem como da Universidade Federal do Amazonas e da Universidade Federal do Ceará para alunos das escolas locais.

Para desenvolvimento da ação na Guiana, foi necessário compreender a realidade da escola local e adaptar os materiais de apresentação existentes. $\mathrm{O}$ material de apresentação foi alterado, mantendo-se apenas os pontos principais - como métodos de entrada, informações gerais dos cursos, bolsas e auxílios - e adicionando-se informações extras - como cursos de Língua Portuguesa para estrangeiros e as formas de ingresso na universidade para alunos estrangeiros. Após a adaptação do conteúdo de apresentação, o material foi todo traduzido para o Inglês, uma vez que é a língua oficial do país. A próxima etapa consistiu na escolha de pelo menos um aluno de cada grupo (PET- Engenharia Civil, CAAU e CAEEL da UFRR) que estivesse apto para se preparar e apresentar na Língua Inglesa. Para a realização do projeto foi necessário levar os materiais digitais de apresentação. A Figura 3 ilustra a execução da ação em Lethem. 
Figura 3 - Representantes do CAAU, CAEEL e PET apresentando o Novos Talentos na St Ignatius Highschool - Lethen

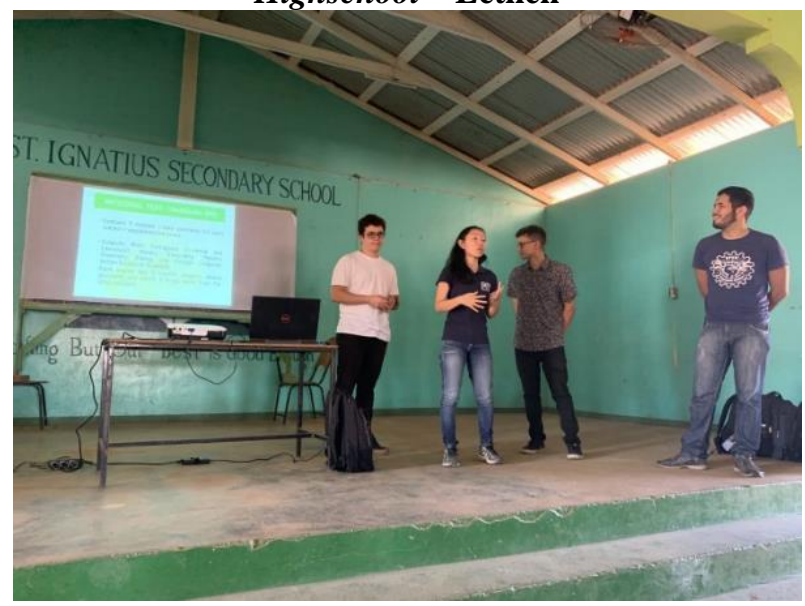

Fonte: arquivo dos autores (2019).

\section{RESULTADOS E DISCUSSÕES}

A princípio, observou-se que grande parte dos estudantes das escolas acompanhadas não apresentavam perspectivas para o ingresso no Ensino Superior e desconheciam não somente os campos de atuação da engenharia, mas também a possibilidade e o direito de ingressar em uma universidade pública gratuita. Tal fato também foi constatado em outras edições do Novos Talentos, o que torna evidente a carência e a necessidade de levar o conhecimento para os estudantes da rede pública.

Nesse sentido, em paralelo às edições de 2017 e 2018, houve uma expansão em relação às localidades de atendimento do projeto, para que escolas, além das do munícipio de Boa Vista, pudessem ser contempladas, abrangendo municípios próximos à capital do estado e até mesmo Lethem, cidade da Guiana. Ao todo seis escolas foram atendidas em 2019, em contraste ao número de três e de cinco escolas nas edições de 2017 e 2018, respectivamente.

Em relação ao número de alunos em 2019, a ação pôde atender uma quantidade significativa, totalizando 419 alunos. Dessa forma, desde o início do projeto Novos Talentos, 1465 alunos foram atendidos pela ação, com perspectivas de abranger um público maior em próximas edições. O gráfico da Figura 4 apresenta as localidades e a quantidade do público atendido na edição de 2019.
Figura 4 - Quantidade de alunos atendidos por localidade em 2019

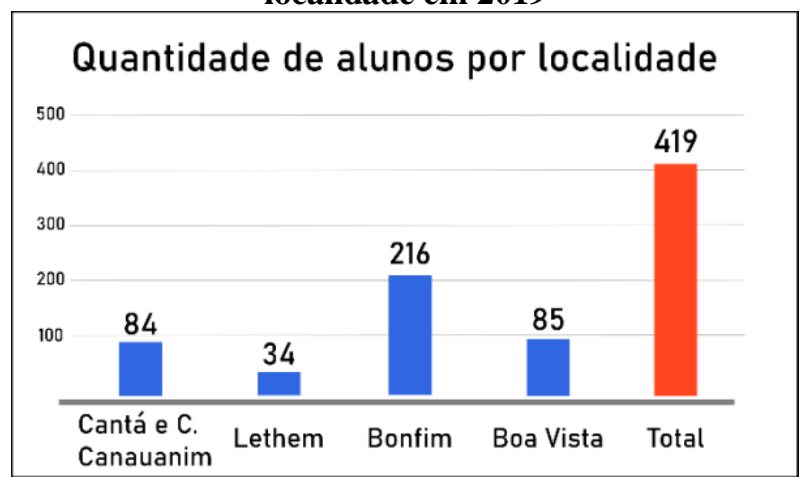

Fonte: arquivo dos autores (2019).

Por conta das novas localidades e da nova faixa etária abordada (Ensino Fundamental), a maneira de apresentação foi modificada para melhor atender às realidades existentes em cada local. Com as modificações, houve um maior envolvimento por conta dos alunos, em especial na Comunidade Indígena Canauanim, onde, durante a dinâmica, os alunos se mostraram bastante engajados e participativos. Nessa ação, foi possível constatar as diferentes reações às atividades apresentadas, onde alguns alunos levaram como brincadeira e diversão; em contrapartida, outros demonstraram maior caráter de competição, mostrando criatividade e capacidade de liderança, ilustrados na Figura 5.

Figura 5 - Engajamento dos alunos na dinâmica realizada pelo grupo PET na comunidade Indígena Canauanim

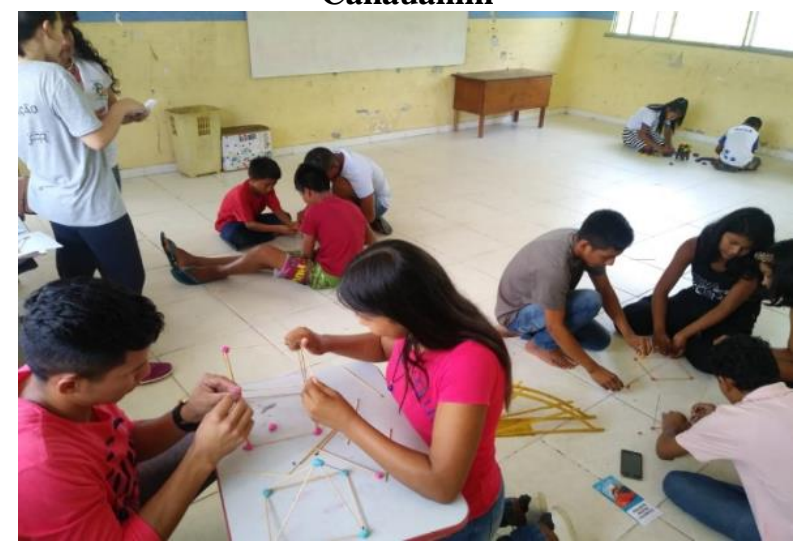

Fonte: arquivo dos autores (2019).

Cabe ressaltar que a nova maneira de abordagem executada na comunidade Canauanim, em que o projeto foi apresentado de forma rotativa, mostrou-se eficiente pois tornou possível uma maior atenção para os alunos e uma maior proximidade, garantindo 
assim o desenvolvimento de uma dinâmica mais participativa e descontraída.

Apesar das mudanças realizadas, a apresentação do projeto foi feita de forma clara aos alunos, mostrando-se as áreas de atuação do profissional, os diversos processos de entrada na UFRR, assim como os auxílios e bolsas oferecidos por diversos programas existentes na instituição, incentivando a permanência do acadêmico durante o seu período de formação.

O projeto representou um passo importante na criação de vínculos com as instituições de ensino visitadas, sejam elas subordinadas ao Governo Estadual, Governo Federal ou mesmo de outro país, como foi o caso da escola na Guiana (Figura 6). Dessa forma, foram favorecidas novas oportunidades de retorno a essas localidades e possíveis aprimoramentos do projeto, pois há uma melhor contrapartida da instituição.

Figura 6 - Apresentação na escola St. Ignatius Highschool, localizada na Guiana.

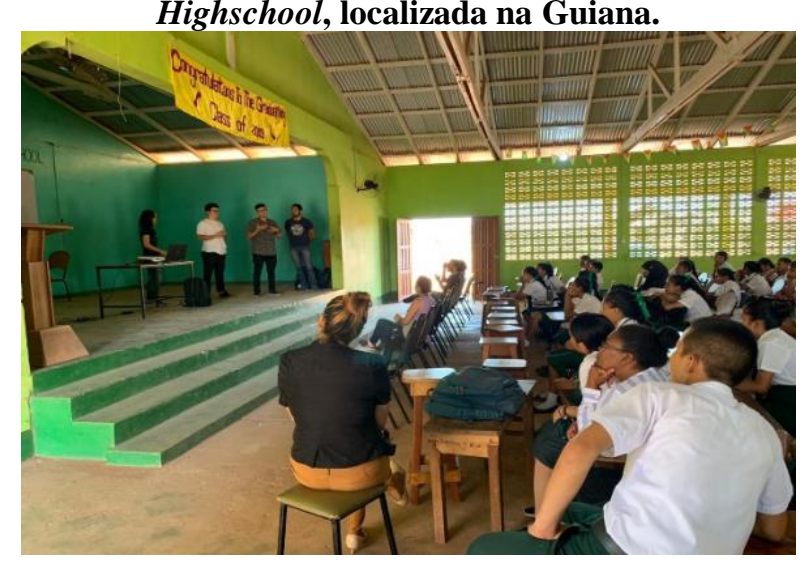

Fonte: arquivo dos autores (2019).

Outro passo importante na edição de 2019 foi o das associações com os Centros Acadêmicos (CAAU e CAEEL). Houve benefícios tanto para os alunos quanto para os integrantes dos Centros Acadêmicos e do PET, pois a parceria possibilitou uma maior diversificação dos assuntos abordados nas apresentações, relatos sob diferentes pontos de vista e geração de conhecimento sob as diferentes percepções.

Com relação à participação dos alunos, em geral, foi observado que eles se envolveram ativamente por meio de perguntas aos integrantes do grupo PET e dos Centros Acadêmicos, além de demonstrarem engajamento nas atividades desenvolvidas. Em acréscimo, é válido comentar que o conteúdo apresentado foi bem recebido pelas crianças e jovens de todas as escolas, gerando debates, direcionando-os e motivando-os a cursarem o Ensino Superior na UFRR.

Atingindo uma quantidade expressiva de alunos das escolas visitadas, a edição de 2019 do projeto foi concluída com sucesso, repassando as informações de forma clara, estimulando e orientando os alunos na sua escolha profissional, através de apresentações expositivas, recursos multimídia, dinâmicas práticas e material impresso. Cabe enfatizar que foi apresentado um leque de oportunidades profissionais ofertadas na universidade, além da dinâmica universitária e das áreas de atuação da Engenharia Civil, Engenharia Elétrica e da Arquitetura e Urbanismo. Apresentou-se também o Programa de Educação Tutorial (PET), com seus objetivos e atuação, assim como os Centros Acadêmicos. Dessa maneira, atrai-se um público mais instruído acerca das oportunidades presentes dentro da universidade pública, consequentemente, menos propenso a entrar nas estatísticas de evasão.

\section{CONCLUSÕES}

O Projeto Novos Talentos cresceu de modo significativo, em relação ao que se projetava para sua realização ao longo dos últimos anos. No que tange ao seu impacto social e ao alcance de alunos de diferentes localidades em estado de vulnerabilidade social, o projeto teve êxito na sua execução.

Objetivando atuar como uma medida de democratização do acesso ao Ensino Superior, ao apresentar a possibilidade de prosseguimento dos estudos para os alunos atendidos, a ação mostrou-se necessária e seu dinamismo satisfatório, sobretudo nas localidades mais afastadas da capital do estado ou de difícil acesso, como o país fronteiriço Guiana e a comunidade indígena Canauanim.

$\mathrm{O}$ engajamento dos alunos atendidos pelo projeto se tornou um parâmetro de avaliação da ação. $\mathrm{O}$ envolvimento ativo dos participantes esteve presente em todas as escolas visitadas, tendo o conteúdo sido bem recebido por todas 
as crianças e jovens. Dessa forma, o Novos Talentos despertou o interesse de ingresso em um Curso Superior, forneceu conhecimento das oportunidades que a graduação traz e expôs os auxílios que a universidade concede ao estudante em vulnerabilidade social, o que possibilita a sua permanência na universidade.

O projeto deu ainda um passo importante na criação de vínculos com as instituições de ensino visitadas, diminuindo a distância entre Universidade e comunidade. Tal fato favorece novas oportunidades de retorno às localidades, com uma melhor contrapartida das instituições, o que possibilita o crescimento e melhoramento da ação, abrindo assim um espaço que facilita a criação de novos projetos que atendam em diferentes âmbitos esse público pouco assistido pela sociedade.

\section{AGRADECIMENTOS}

Os autores agradecem ao Ministério da Educação pela oportunidade de realizarem projetos de extensão e pesquisa por meio do grupo PET; ao Fundo Nacional de Desenvolvimento e Educação (FNDE) pelo apoio financeiro; ao Departamento de
Engenharia Civil pelo apoio aos trabalhos desenvolvidos pelo grupo.

\section{REFERÊNCIAS}

ESBELL, V. F. et al. Projeto Novos Talentos: uma nova perspectiva sobre a universidade. Anais... XLVII Congresso Brasileiro de Ensino de Engenharia, 2019, Fortaleza. Fortaleza: COBENGE, 2019.

FREITAS, M. G. P. et al. Os desafios da entrada e permanência na universidade por estudantes da classe trabalhadora. Anais... XXI Encontro Latino Americano de Iniciação Cientifica, Univap, 2017, São José dos Campos, SP. São José dos Campos: INIC, 2017.

ORTEGA, E. M. V. O ensino médio público e o acesso ao ensino superior. Estudos em avaliação educacional, São Paulo, p. 1-24, 2001.

SAMPAIO, S. M. R. (Org.). Entre a escola pública e a universidade: longa travessia para jovens de origem popular. Observatório da vida estudantil: primeiros estudos. Salvador: EDUFBA, 2011, p. 27-51.

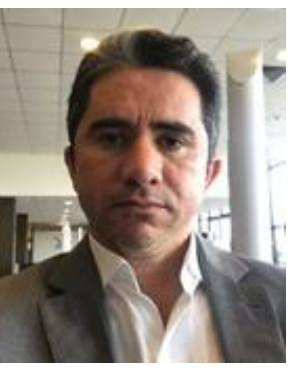

\section{DADOS BIOGRÁFICOS DOS AUTORES}

Adriano Frutuoso da Silva - Matemático pela Universidade Estadual da Paraíba UEPB (1998), Engenheiro Civil pela Universidade Federal da Paraíba - UFPB (2000). Mestre (2003) e Doutor (2007) em Geotecnia pela Universidade de Brasília. Professor Substituto da Universidade de Brasília (2006-2007). Professor Adjunto da Sociedade Unificada Paulista de Ensino Renovado Objetivo/UNIP/Campus de Brasília (2004-2005). Professor Adjunto do Centro de Estudo Superior Planalto/IESPLAN/Brasília (2009). Analista de Projetos de Pavimentação - Siscon Consultoria de Sistema Ltda/DNIT(2007). Coordenador de Projetos de PCH e UHE - Larrosa \& Santos Consultores Associados Ltda (2007-2009). Professor Adjunto do Curso de Engenharia Civil da Universidade Federal de Roraima (2009-2013). Professor Adjunto do Departamento de Engenharia Hidráulica e Ambiental da Universidade Federal do Ceará (2013-2016). Professor do Programa de PósGraduação em Engenharia Civil, POSDEHA/UFC (2013-2016). Atualmente Professor Associado do Curso de Engenharia Civil da Universidade Federal de Roraima, Professor do Programa de Pós-Graduação ProfÁgua/UFRR e do Programa de Pós-Graduação em Engenharia de Barragens e Gestão Ambiental PEBGA/UFPA. Tutor do Programa Tutorial - PET Engenharia da UFRR (20172022). Presidente do Núcleo Região Norte da ABMS (2019-2020). Presidente do Núcleo Região Norte do CBDB (2020). Tem experiência na área de Engenharia Civil, atuando nos seguintes temas: Obras de Terra e Enrocamento, Modelagem 
Numérica de Obras Geotécnicas, Geotecnia Ambiental e Regulação e Governança de Recursos Hídricos.

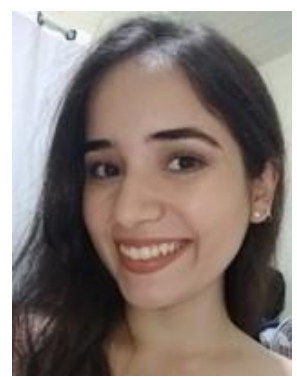

Camila Helena Menezes de Oliveira - Acadêmica do $9^{\circ}$ semestre do curso de Engenharia Civil na Universidade Federal de Roraima (UFRR). Participa do Programa de Educação Tutorial, PET-Engenharia Civil da UFRR.

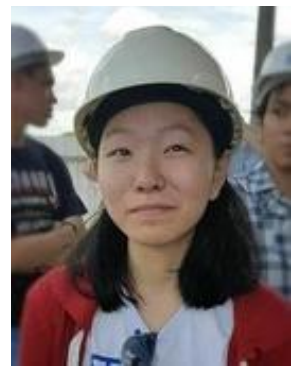

Danielle Yumi Mizuno - Acadêmica do $9^{\circ}$ semestre do curso de Engenharia Civil na Universidade Federal de Roraima (UFRR). Participa do Programa de Educação Tutorial, PET-Engenharia Civil da UFRR.

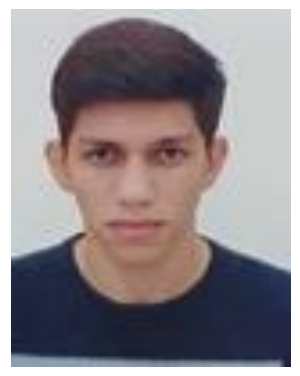

Felipe de Melo Medeiros - Acadêmico do $6^{\circ}$ semestre do curso de Engenharia Civil na Universidade Federal de Roraima (UFRR). Participa do Programa de Educação Tutorial, PET-Engenharia Civil da UFRR.

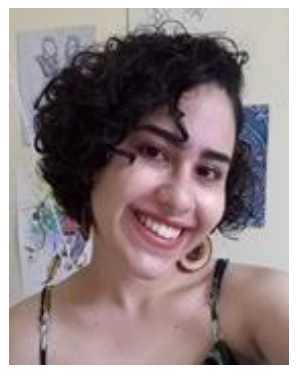

Hingred Karoline Magalhães da Luz - Acadêmica do $6^{\circ}$ semestre do curso de Engenharia Civil na Universidade Federal de Roraima (UFRR). Participa do Programa de Educação Tutorial, PET-Engenharia Civil da UFRR.

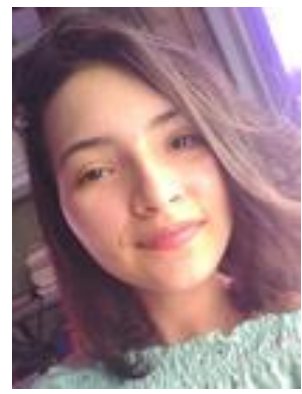

Rebeca Dias de Souza - Acadêmica do $6^{\circ}$ semestre do curso de Engenharia Civil na Universidade Federal de Roraima (UFRR). Participa do Programa de Educação Tutorial, PET-Engenharia Civil da UFRR. 


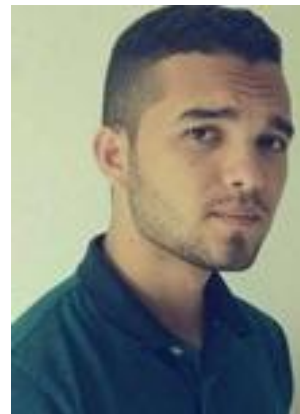

Rones de Souza Santos - Acadêmico do $9^{\circ}$ semestre do curso de Engenharia Civil na Universidade Federal de Roraima (UFRR). Participa do Programa de Educação Tutorial, PET-Engenharia Civil da UFRR. 